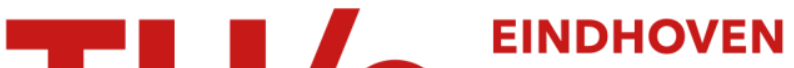

\section{Relationship between electrohysterogram and internal uterine pressure: a preliminary study}

\section{Citation for published version (APA):}

Rabotti, C., Mischi, M., Laar, van, J. H., Aelen, P., Oei, S. G., \& Bergmans, J. W. M. (2006). Relationship between electrohysterogram and internal uterine pressure: a preliminary study. In Proceedings 200628 th Annual International Conference of the IEEE Engineering in Medicine and Biology Society : New York, NY, 30 August - 3 September 2006 (pp. 1661-1664). Institute of Electrical and Electronics Engineers. https://doi.org/10.1109//EMBS.2006.259928

DOI:

10.1109/IEMBS.2006.259928

Document status and date:

Published: 01/01/2006

\section{Document Version:}

Publisher's PDF, also known as Version of Record (includes final page, issue and volume numbers)

\section{Please check the document version of this publication:}

- A submitted manuscript is the version of the article upon submission and before peer-review. There can be important differences between the submitted version and the official published version of record. People interested in the research are advised to contact the author for the final version of the publication, or visit the $\mathrm{DOI}$ to the publisher's website.

- The final author version and the galley proof are versions of the publication after peer review.

- The final published version features the final layout of the paper including the volume, issue and page numbers.

Link to publication

\section{General rights}

Copyright and moral rights for the publications made accessible in the public portal are retained by the authors and/or other copyright owners and it is a condition of accessing publications that users recognise and abide by the legal requirements associated with these rights.

- Users may download and print one copy of any publication from the public portal for the purpose of private study or research.

- You may not further distribute the material or use it for any profit-making activity or commercial gain

- You may freely distribute the URL identifying the publication in the public portal.

If the publication is distributed under the terms of Article 25fa of the Dutch Copyright Act, indicated by the "Taverne" license above, please follow below link for the End User Agreement:

www.tue.nl/taverne

Take down policy

If you believe that this document breaches copyright please contact us at:

openaccess@tue.nl

providing details and we will investigate your claim. 


\title{
Relationship between electrohysterogram and internal uterine pressure: a preliminary study
}

\author{
C. Rabotti*, M. Mischi*, J. O. E. H. van Laar**, P. Aelen*, S. G. Oei**, and J. W. M. Bergmans*
}

\begin{abstract}
Electrohysterography (EHG) is a promising technique for monitoring the uterine activity, based on electrical recordings on the abdominal surface. However, a quantitative estimation of the internal uterine pressure (IUP) by means of EHG is not available for clinical practice. In this paper we present a preliminary study on the estimation of the mechanical uterine activity from abdominal EHG measurements. For the EHG analysis we use two different Time Frequency Distributions (TFD): the spectrogram and the Wigner-Ville Distribution. We assume the EHG to be the sum of frequency modulated signals. Based on this assumption, the IUP is estimated from the unnormalized first moment of the TFD. Eventually, a third order polynomial model is applied to the estimated IUP in order to improve the estimate accuracy. A recent method for detection of uterine contraction by EHG is employed for comparison of the performance. The algorithms were tested on two patient recordings. The results were compared with a reference IUP which is simultaneously measured by an intrauterine catheter. The IUP estimated by our method showed a correlation coefficient with the reference IUP $(R=0.93)$ higher than that achieved by the comparison method $(R=0.85)$. Therefore the proposed method may be considered as a promising clinical technique for accurate non invasive IUP measurements.
\end{abstract}

\section{INTRODUCTION}

Preterm birth is a major factor for long-term morbidity and accounts for between $69 \%$ and $85 \%$ of neonatal deaths not caused by congenital malformations [1]. One of the keys to treat preterm labor is early detection and prediction by monitoring the uterine activity.

Besides the more traditional methods employing pelvic examination and symptomatic self monitoring, tocography is currently the most widely used technique to monitor the activity of the uterus during pregnancy and parturition [2]. The primary advantage of a tocodynamometer consists of its non invasiveness. Nevertheless, tocography is affected by a high level of inaccuracy. Additionally, the use of tocodynamometer can only provide information related to the frequency of the contractions and does not involve any direct or indirect measure of uterine functionality, such as force or efficiency of the contractions.

Quantitative information concerning uterine contraction can be provided by measuring the internal uterine pressure (IUP) with an intrauterine catheter. However, this technique requires the rupture of the membranes and, due to its high invasiveness, it can increase the risk of infections.

This work was supported by the Dutch Technology Fundation, STW

* C. Rabotti, M. Mischi, P. Aelen, and J. W. M. Bergmans are with the department of Electrical Engineering, University of Technology Eindhoven, Eindhoven, the Netherlands.

**J. O. E. H. van Laar and S. G. Oei are with the Máxima Medisch Centrum, Veldhoven, The Netherlands.
Electrohysterography (EHG) records the bioelectrical signal associated to muscle contraction. Therefore, it is an alternative non-invasive method to monitor the uterine activity. It has been demonstrated that the uterine electromyographic signal originated from action potentials can be measured from the mother's abdomen with contact electrodes [3]. The action potentials are strictly related to the contractile functions of the muscle. Synchronization between mechanical activity and electrical abdominal signal has been extensively demonstrated in literature, in particular, an increase of the IUP is the result of the simultaneous contraction of an adequate number of cells. However, a mathematical description of the relationship between the electrohysterographic signal recorded from the abdomen and the IUP during pregnancy and parturition is not yet available. The availability of this mathematical description could allow non invasive IUP assessment.

In this paper we investigate the relationship between the uterine electrohysterographic signal measured on the mother abdomen and the IUP simultaneously measured with an intrauterine catheter during non active labor. The aim of this study is the development of a mathematical model for the estimation of the IUP from electrohysterographic recordings. The EHG signal is analyzed by means of two Time-Frequency distributions (TFD): the spectrogram and the Wigner-Ville Distribution (VWD). Differently from [4], where the EHG is considered as originated by a single FM process, we regard the EHG as originated by a summation of FM signals related to the IUP. In fact, because the EHG is recorded by surface electrodes, it is the result of a spatial summation of action potentials from different origins. Therefore, the unnormalized first moment of the TFD in a selected frequency band is used to estimate the instantaneous frequency and detect the onset of contractions. Eventually, a polynomial model is proposed to minimize the mean square error between the estimated IUP and the IUP measured with the catheter. In this preliminary study a third order polynomial model is adopted with promising results. The only method found in literature for a quantitative analysis of contraction patterns is described in [5]. This method, which is based on the calculation of the RMS value of the EHG signal in a selected frequency band, is used to validate our method by comparison of the performance. The IUP estimated by our method showed a correlation coefficient with the reference IUP $(R=0.93)$ higher than that achieved by the comparison method $(R=0.85)$, confirming the better performance of our algorithm and the possibility of non invasive IUP assessment by EHG. 


\section{METHODOLOGY}

\section{A. Experimental set-up}

Our method has been tested on 2 measurements recorded on two women during non active labor at the Máxima Medical Center in Veldhoven (The Nethelands). Each recording consists of about 15 minutes of data. The EHG was measured by means of 11 unipolar contact $\mathrm{Ag}-\mathrm{AgCl}$ electrodes placed on the mother's abdomen as shown in Fig. 1. The reference for the electrodes was on the right mother's ankle. This configuration is chosen to cover as much of the uterus as possible and, eventually, to determine the electrode position that shows the largest signal-to-noise ratio (SNR). An intrauterine pressure catheter Koala M1333A (Philips) was inserted in the uterine cavity for the IUP measurement. The IUP and the EHG are simultaneously recorded and digitized at a sampling rate of $500 \mathrm{~Hz}$ with a Maastricht-Programmable AcQuisitionsystem (M-PAQ).
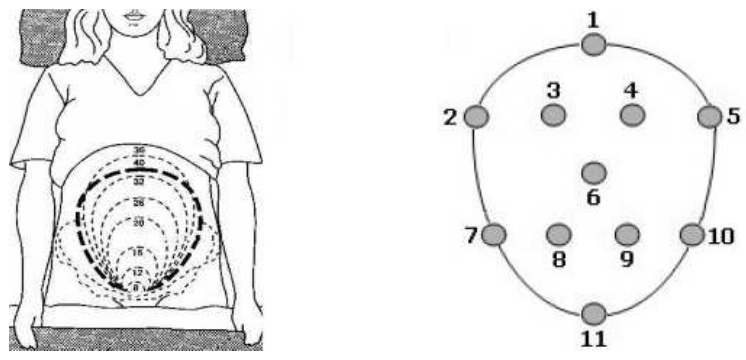

Fig. 1. Electrode configuration

\section{B. Signal pre-processing}

The unipolar recordings are made bipolar digitally, by subtracting the signal of contiguous electrodes. Bipolar electrodes reflect the differences between the two signals present below the electrodes. Therefore, in most cases the common noise such as the maternal electrocardiogram, maternal movements, and power line interference may be efficiently rejected [6]. Furthermore, as the uterine electrohysterogram frequency content ranges between 0 and $5 \mathrm{~Hz}$ [6], the digital analysis of the signal can be performed at a sampling frequency close to $10 \mathrm{~Hz}$. Therefore, the acquired signal is downsampled by a factor 5, after applying an atialiasing Finite Impulse response (FIR) filter with a cut-off frequency at $5 \mathrm{~Hz}$.

\section{Signal analysis}

The measured EHG signal is not stationary. An ordinary Fourier Transform integrates all the frequency components over the whole observation interval. Because of this averaging, the time discrimination is lost. The use of a TimeFrequency Distribution (TFD) is a proper solution to this problem.

Since we measure electrical energy, which is a quadratic signal, the class of quadratic TFDs is used. The TFD $\rho_{z}(t, f)$ can be seen as the Fourier transform in the lag variable $\tau$ of a weighted non stationary autocorrelation [7]:

$$
\begin{array}{r}
\rho_{z}(t, f)=\int_{-\infty}^{+\infty} \int_{-\infty}^{+\infty} \int_{-\infty}^{+\infty} g(v, \tau) z\left(t+\frac{\tau}{2}\right) z^{*}\left(t-\frac{\tau}{2}\right) \times \\
e^{j 2 \pi(v t-v u-f \tau)} d u d v d \tau,
\end{array}
$$

where the Doppler-lag kernel $g(v, \tau)$ defines a frequency weighting and time averaging function. The signal $z(t)$ is the analytic associate of the measured signal $s(t)$, i.e., the negative part of the frequency spectrum is removed to suppress cross terms in $\rho_{z}(t, f)$. The Doppler-lag signal kernel $g(v, \tau)$ determines the main properties of the resulting TFD. In this study two different signal kernels are used. The first kernel is simply $g(v, \tau)=1$. The use of this kernel results in the Wigner-Ville Distribution (WVD) as given in (2):

$$
\rho_{z}(t, f)=\int_{-\infty}^{+\infty} z\left(t+\frac{\tau}{2}\right) z^{*}\left(t-\frac{\tau}{2}\right) e^{-j 2 \pi f \tau} d \tau .
$$

The second one is the signal kernel $g(v, \tau)=\int_{-\infty}^{+\infty} w(t+$ $\left.\frac{\tau}{2}\right) w\left(t-\frac{\tau}{2}\right) e^{-j 2 \pi v t} d t$, which results in the widely used spectrogram as given in (3):

$$
\rho_{z}(t, f)=\left|\int_{-\infty}^{+\infty} z(\tau) w(\tau-t) e^{-j 2 \pi f \tau} d \tau\right|^{2}
$$

The choice of the spectrogram involves a compromise between time and frequency resolution. In our measurements a window of 40 seconds provides the best results in terms of IUP estimation.

\section{Instantaneous frequency}

The uterine EMG can be described as a series of action potentials, whose frequency can be related to the phase of the mechanical activity. These variations can be seen as a frequency modulation phenomenon where the modulating signal is related to the evolution of the IUP. However, the EHG is not a clear mono component signal, and the signal in each electrode can be viewed as the result of a sum of FM signals. Therefore, a measure of contractile strength, i.e., the instantaneous frequency, can be provided by the average frequency. This average frequency is calculated by the first moment in frequency domain of $\rho(t, f)$ as given in (4):

$$
\Omega(t)=\frac{\int_{-\infty}^{+\infty} f \rho(t, f) d f}{\int_{-\infty}^{+\infty} \rho(t, f) d f} .
$$

Because of the spatial integration of FM signals, also the amplitude of the EHG contains information about the IUP. The TFD integrated in frequency provides the instantaneous power as given in (5):

$$
p(t)=\int_{-\infty}^{+\infty} \rho(t, f) d f .
$$


By multiplying equation (4) and (5) a new feature $\psi(t)$ is obtained with information about both amplitude and frequency of $\rho(t, f)$ :

$$
\psi(t)=\Omega(t) \cdot p(t)=\int_{f_{\min }}^{f_{\max }} f \rho(t, f) d f .
$$

The feature $\psi(t)$ obtained by (6) is the unnormalized first moment in frequency of $\rho(t, f)$, and it will be evaluated to estimate the IUP. The first moment is evaluated in a selected frequency band $\left[f_{\text {min }}, f_{\text {max }}\right]$, which is experimentally determined to maximize the final correlation coefficient with the reference IUP. The frequency range in which the best results are obtained, differs for the two TFDs. For the WVD the frequency range from $0.7 \mathrm{~Hz}$ to $2.4 \mathrm{~Hz}$ gives the best results and for the spectrogram this range goes from $0.2 \mathrm{~Hz}$ to $1.1 \mathrm{~Hz}$, independently from the considered electrode. Nevertheless, the performance of the WVD is less influenced by the chosen frequency band.

\section{E. Multiple regression}

The unnormalized first moment obtained with the described algorithm can be further processed in order to increase the accuracy of the estimated IUP. To this end, we adopted a model which takes into account the offset, gain, and non linear effects describing the relationship between the electrical and the mechanical activity of the uterus. Two different factors can cause these non linearities: the local meaning of electrical recordings and the possible consequences of muscle fatigue. The first factor can be explained considering that each electrode records the electrical activity localized below or in the neighborhood of the electrode position. Nevertheless, the IUP increase is the result of the simultaneous contraction of an adequate number of cells, and it is associated to a widespread electrical activity of the whole uterine muscle. Therefore, localized or unsynchronized electrical activities can be recorded by the neighboring electrodes without being necessarily followed by a linearly related IUP increase. The second factor which can cause a non linear relation between mechanical and electrical uterine activity is muscle fatigue. In fact, it is plausible, due to muscle fatigue during labor, that the production of lactate inhibits a proper muscle reaction to the electrical activation.

We indicate by $\Psi_{k}=\left(\psi\left(t_{1}\right), \psi\left(t_{2}\right), \ldots, \psi\left(t_{N}\right)\right)$ the digitized instantaneous frequency calculated in each channel $k$ and by $\underline{Y}=\left(y\left(t_{1}\right), y\left(t_{2}\right), \ldots, y\left(t_{N}\right)\right)$ the sampled IUP measured by the catheter. Due to the propagation delay of the electric signal through the electrodes, the instantaneous frequency is first temporally aligned to $\underline{Y}$ by maximization of the crosscorrelation function. After alignment, the IUP is represented by a polynomial expansion of $\underline{\Psi}$ with coefficients $a, b, c$, and $d \in R$ as given in (7):

$$
\underline{\hat{Y}_{k}}=a+b \underline{\Psi_{k}}+c \underline{\Psi_{k}^{2}}+d \underline{\Psi_{k}}{ }^{3} .
$$

In (7) $a$ models the offset, $b$ the gain, and $c$ and $d$ the nonlinearities of the relationship between the estimated IUP and the reference IUP in each channel $k$.
The values of $a, b, c$ and $d$ are estimated by a least squares method as given in (8), where $\varepsilon_{k}$ is the squared error to be minimized and $\mathrm{N}$ is the number of samples:

$$
\varepsilon_{k}=\sum_{i=1}^{N}\left(y\left(t_{i}\right)-\left[a+b \psi\left(t_{i}\right)+c \psi\left(t_{i}\right)^{2}+d \psi\left(t_{i}\right)^{3}\right]\right)^{2} .
$$

By defining the matrix

$$
\mathbf{X}=\left[\begin{array}{cccc}
1 & \psi\left(t_{1}\right) & \psi\left(t_{1}\right)^{2} & \psi\left(t_{1}\right)^{3} \\
1 & \psi\left(t_{2}\right) & \psi\left(t_{2}\right)^{2} & \psi\left(t_{2}\right)^{3} \\
\vdots & \vdots & \vdots & \vdots \\
1 & \psi\left(t_{N}\right) & \psi\left(t_{N}\right)^{2} & \psi\left(t_{N}\right)^{3}
\end{array}\right]
$$

and parameter vector $\underline{P}=\left[\begin{array}{llll}a & b & c & d\end{array}\right]$, the optimal least squares solution [8] is found by (9):

$$
\underline{\hat{P}}=\left(\mathbf{X}^{t} \mathbf{X}\right)^{-1} \mathbf{X}^{t} \underline{Y}
$$

If the value of $\underline{\hat{P}}$ is consistent through all the measurements, it can be used to improve modeling and estimation of IUP from EHG signals.

\section{RESULTS AND DISCUSSION}

The IUP was estimated with the proposed method in each bipolar channel. The quality of the estimation is assessed by calculating the correlation coefficient $(R)$ with the IUP simultaneously measured by the intrauterine catheter. Our method is validated by comparing its performance with a reference method [5]. With the proposed method, a good estimation of the IUP can be obtained by almost all the electrodes, as long as they are above the corpus uteri. Nevertheless, electrode placement along the the vertical median axis is likely to provide a better SNR because of a closer contact and a more constant position of the uterus relative to the abdominal wall during contractions [6]. In our recordings, the best results are obtained in the electrodes in the lower vertical median axis of the abdomen. In particular, the final results showed in Fig. 2, Fig. 3, and Fig. 4 are obtained by the same bipolar electrode (9-6). This demonstrates that the use of a subset of electrodes is sufficient. Additionally, though both the spectrogram and WVD provide a high correlation with the reference IUP, the WVD gives a better signal when there is no contraction (Fig. 3), and it is therefore to be preferred. The results in terms of correlation coefficient are shown in Table I for the bipolar electrode 9-6. The reference algorithm results in the poorest estimates.

A preliminary evaluation of the coefficients of the polynomial model was performed on the two available measurements during non active labor. In the examined cases, $c$ and $d$, i.e., the coefficients of the non-linear terms, are negligible and polynomial models of the second and third order provide similar error $\varepsilon_{k}$. This result was expected due to the early stage of labor of the two examined cases. At this stage, in fact, the electrical activity of the uterus is well established and sufficiently coordinated to produce efficient contractions, i.e., each detected electrical burst is followed by a proportional IUP increase. Moreover, muscular fatigue is not expected at this early stage of labor. We could however 


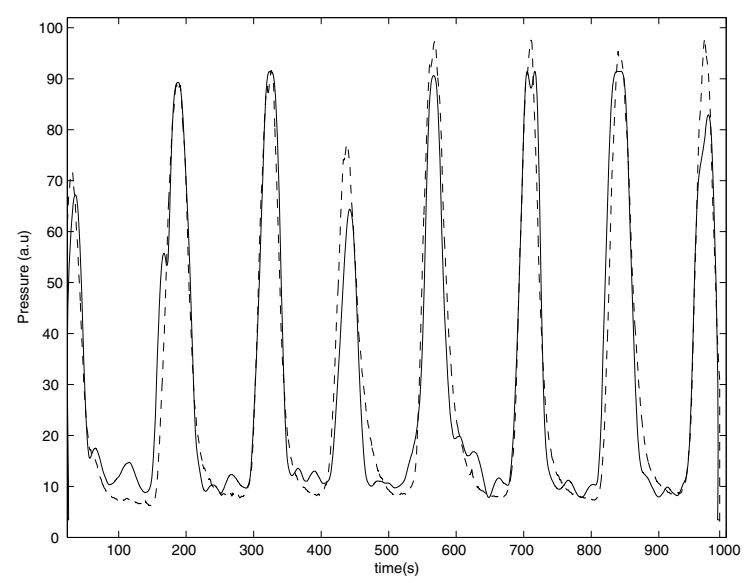

Fig. 2. Dashed line: IUP measured by the catheter. Solid line: IUP estimated by our method in the bipolar electrode 9-6 using the spectrogram.

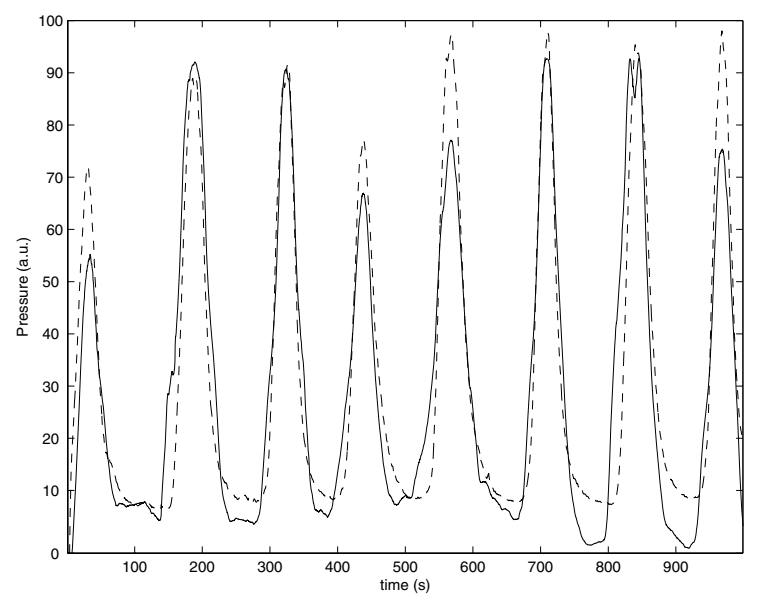

Fig. 3. Dashed line: IUP measured by the catheter. Solid line: IUP estimated by our method in the bipolar electrode 9-6 using the WVD

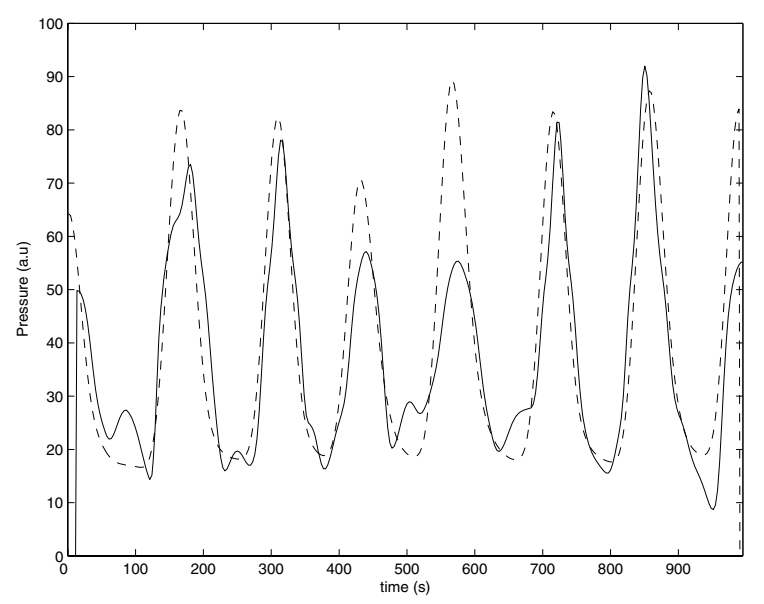

Fig. 4. Dashed line: IUP measured by the catheter. Solid line: IUP estimated by the algorithm [5] in the bipolar electrode 9-6. expect the non linear terms of the polynomial model to be more significant during pregnancy, or in more advanced stages of labor.

TABLE I

CORRELATION WITH THE REFERENCE IUP

\begin{tabular}{c|lc}
\hline Method & \multicolumn{2}{|c}{ Correlation coefficient $R(p)$} \\
\hline Spectrogram & 0.93 & $(<0.001)$ \\
Wigner Ville & 0.88 & $(<0.001)$ \\
Reference & 0.85 & $(<0.001)$ \\
\hline
\end{tabular}

\section{CONCLUSIONS AND FUTURE WORKS}

In this paper we describe a preliminary study on the relationship between the uterine mechanical activity as recorded by an intra-uterine catheter and the mechanical activity estimated by processing the electrohysterographic signal recorded from the abdominal surface. A novel approach is proposed for the estimation of the IUP and a polynomial model is adopted to optimize the estimate. The method produces accurate results with a correlation coefficient $R=0.93$. These promising results suggest the possibility to propose EHG as a valid alternative to the existing techniques, which can potentially combine the non invasiveness of tocographic measurement with the accuracy of an IUP measurement device. In the two examined cases during non active labor, the relationship between the reference and estimated IUP is well approximated by a linear relationship. Nevertheless, we expect this relation to be non linear in case of non-efficient contractions or in case of muscle fatigue. The TFDs that are used in this study are the WVD and the spectrogram, where the results for the WVD are slightly better. Future work will involve the investigation of other time frequency techniques and, if required, the development of a signal dependent kernel. In general, extensive experimentation will be necessary to broadly validate our method and to determine the parameters of our polynomial model.

\section{REFERENCES}

[1] G. S. Berkowitz and E. Papiernik , "Epidemiology of preterm birth", Epid. Rev., vol. 15, 1993, pp 414-443.

[2] R. E. Garfield, H. Maul, W. Maner, C. Fittkow, G. Olson, L. Shi, and G. R. Saade, "Uterine electromyography and light-induced fluorescence in the management of term and preterm labor", J. Soc. Gynecol. Investig., vol. 9, 2002, pp 265-275.

[3] C. Buhimschi and R. E. Garfield , "Uterine contractility as assessed by abdominal surface recording of electromyografic activity in rats during pregancy", Am. J. Obstet. Gynecol., vol. 174, 1996, pp 744-753.

[4] S. Mansour, D. Devedeux, J. Duchêne, G. Germain, and C. Marque, "Uterine EMG spectral characteristics and instantaneous frequency measurement", IEEE EMBS Proc., 1992, pp 2602-2603.

[5] J. Jezewski and K. Horoba and A.Matonia and J. Wrobel, "Quantitative analysis of contraction patterns in electrical activity signal of pregnant uterus as an alternative to mechanical approach", Physiol. Meas., vol. 26, 2005, pp 753-767.

[6] D. Devedeux, C. Marque, S. Mansour, G. Germain, and J. Duchêne, "Uterine EMG analysis: a critical review", Am. J. Obstet. Gynecol., vol. 169, 1993, pp 1636-1653.

[7] B. Boashash, Time frequency signal analysis and processing: a comprehensive reference, Elsevier; 2003.

[8] D. G. Manolakis, V. K. Ingle, and S. M. Kogon , Statistical and adaptive signal processing, Artech house, Boston; 2005.

[9] H. Kwakernaak and R. Sivan, Modern signals and systems, Prentice Hall, Englewood Cliffs, NJ; 1991. 